\title{
(Digital) PhotoVoice y su papel en el Desarrollo Comunitario y la construcción de la identidad colectiva: hacia una ciudadanía crítica y participativa a través de la educación
}

The role of (Digital) PhotoVoice in Community Development and the construction of collective identity: promoting critical and participative citizens through education

(Digital) PhotoVoice i el seu paper en el Desenvolupament Comunitari i la construcció de la identitat col-lectiva: cap a una ciutadania crítica i participativa a través de l'educació

José Luis Fernández-Pacheco Sáez ${ }^{1, *}$, Irina Rasskin Gutman ${ }^{1}{ }^{\circledR}$, Eduardo Marques $^{2 \odot}$, Mieko Yoshihama $^{3 \odot}$

\section{Cómo citar:}

Fernández-Pacheco Sáez,

J.L., Rasskin-Gutman, I.,

Marques, E. y Yoshihama, M. (2022). (Digital)

PhotoVoice y su papel en el Desarrollo Comunitario y la construcción de la identidad colectiva: hacia una ciudadanía crítica y participativa a través de la educación. Research in Education and Learning Innovation Archives, 28,77-93.

$10.7203 / 28.21795$

Copyright: El/La Autor/a. Open Access: Este es un artículo de acceso abierto distribuido bajo los términos de la licencia Creative Commons

Attribution-NoDerivatives 4.0 International licence (CC BY-ND 4.0)

Financiación: Algunas de las experiencias prácticas recogidas en este artículo forman parte de la metodología empleada en el proyecto ESCUTA, financiado por Erasmus + (Código: 2020-1-PT01-KA203078639)
1 | Departamento de Psicología y Antropología, Universidad de Extremadura, España

2 | Faculdade de Ciencias Sociais e Humanas, University of Azores, Ponta Delgada, Portugal

3 | School of Social Work, University of Michigan, Estados Unidos de América

*Autor para correspondencia: jlfernandezps@unex.es (José Luis Fernández-Pacheco Sáez)

Recibido: 22/10/2021 | Aceptado: 20/12/2021 | Publicado: 15/01/2022

RESUMEN: Las sociedades que conforman el planeta, cada vez más globalizadas, requieren la búsqueda del equilibrio entre la poderosa transformación desencadenada por la tecnología digital y la necesidad de conformar sociedades en las que se priorice la igualdad, la justicia, la sostenibilidad ecológica y la resiliencia frente a una crisis sistémica como la actual. Si bien la tecnología digital ha permitido la comunicación y la participación entre segmentos de la sociedad anteriormente aislados y marginados, también ha contribuido a ampliar las brechas de capital social, cultural y financiero. Conscientes de esta realidad, en este trabajo nos centraremos en los aportes que una metodología de Investigación-Acción Participativa como PhotoVoice (Wang y Burris, 1994, 1997) aplicada a un contexto digital -en lo que hemos denominado Digital PhotoVoice (Marques, Fernández-Pacheco, y Yoshihama, en prensa)-, tiene para la dinamización de procesos de Desarrollo Comunitario, de construcción de la identidad colectiva, así como para el fortalecimiento de procesos de resiliencia frente a la crisis. Buscamos, de esta manera, un equilibrio en el que los que los avances y herramientas de la sociedad digital permitan espacios de transformación y reflexión sobre la sostenibilidad y la justicia social. En este artículo presentamos, en primer lugar, las orientaciones teóricas, filosóficas y epistemológicas de PhotoVoice para, en segundo lugar, exponer algunos casos prácticos en los que se está empleando dicha metodología. Basándonos en nuestra propia experiencia y el trabajo de otros autores, examinaremos las posibilidades y desafíos de PhotoVoice en la era digital y su papel para la transformación social.

PALABRAS CLAVES: Digital Photovoice; Participación; Desarrollo Comunitario; identidad colectiva; COVID-19; resiliencia

ABSTRACT: Our increasingly globalized societies require an equilibrium between the powerful transformation released by digital technology and the need to configure societies that prioritize equality, justice, ecological sustainability and resilience when faced with systemic crises such as the one we 
are currently experiencing. Although digital technology has enabled communication and participation between previously isolated and marginalized sectors of society, it has also led to widening gaps in social, cultural and financial capital. Given this reality, in this paper we focus on the contributions a Participatory Action Research methodology such as PhotoVoice (Wang y Burris, 1994, 1997) -when applied to a digital context we call Digital PhotoVoice (Fernández-Pacheco, Rasskin-Gutman, Marques, y Yoshihama, en prensa) - can make, in the context of the current crisis, to stimulate the processes involved in Community Development and the construction of collective identity, and to strengthen the processes of resilience. Our aim is to find spaces for equilibrium where the advances and tools of the digital society enable environments for transformation and reflection on sustainability and social justice. In this article we present the theoretical, philosophical and epistemological orientations of PhotoVoice before discussing several practical cases in which this methodology is used. Drawing on our own experience and those of other authors, we examine the possibilities and challenges presented by PhotoVoice in the digital age and its role in social transformation.

KEYWORDS: Digital PhotoVoice; Participation; Community Development; Collective Identity; Covid-19; Resilience

RESUM: Les societats que conformen el planeta, cada vegada més globalitzades, requereixen la cerca de l'equilibri entre la poderosa transformació desencadenada per la tecnologia digital i la necessitat de conformar societats en les quals es prioritze la igualtat, la justícia, la sostenibilitat ecològica i la resiliència enfront d'una crisi sistèmica com l'actual. Si bé la tecnologia digital ha facilitat la comunicació i la participació entre segments de la societat anteriorment aillats i marginats, també ha contribuït a ampliar les bretxes de capital social, cultural i financer. Conscients d'aquesta realitat, en aquest treball ens centrarem en les aportacions que una metodologia d'investigació-acció participativa com la del PhotoVoice (Wang y Burris, 1994, 1997), aplicada a un context digital -en el que hem denominat PhotoVoice digital (Fernández-Pacheco et al., en prensa) - pot fer a la dinamització de processos de desenvolupament comunitari i construcció de la identitat col-lectiva, així com a l'enfortiment de processos de resiliència enfront de la crisi. Cerquem d'aquesta manera llocs d'equilibri en els quals els avanços i les eines de la societat digital permeten obrir llocs de transformació i reflexió sobre la sostenibilitat i la justícia social. En aquest article presentem en primer lloc les orientacions teòriques i filosòfiques de PhotoVoice, i en segon lloc exposem alguns casos pràctics d'utilització d'aquesta metodologia. Basant-nos en la nostra pròpia experiència i en el treball d'altres autors, examinarem les possibilitats i els desafiaments de PhotoVoice en l'era digital i el seu paper per a la transformació social.

PARAULES CLAU: Digital PhotoVoice; Participació; Desenvolupament Comunitari; Identitat col-lectiva; Covid-19; Resiliència

Notas de aplicación práctica

Qué se sabe sobre el tema

- La metodología PhotoVoice ha sido ampliamente utilizada con muy buenos resultados desde la década de los 90 en ámbitos de la intervención social y de la Cooperación al Desarrollo.

- Sus impulsoras Caroline Wang y Mary Ann Burris siguen siendo un referente importante, aunque la metodología ha ido evolucionando y adaptándose a nuevos contextos y realidades.

- Pese a que tradicionalmente se ha utilizado en procesos de diagnóstico, intervención social y promoción de la salud dentro del marco de la Investigación-Acción Participativa, actualmente su uso se ha ampliado a otros enfoques dentro de la investigación cualitativa, permitiendo nuevas posibilidades de análisis.

\section{Qué añade este trabajo}

- Este trabajo pretende contribuir a la difusión de PhotoVoice como herramienta de transformación social en diferentes ámbitos, al tiempo que pretende reflexionar sobre su adaptación a la Sociedad Digital en lo que denominamos "Digital PhotoVoice".

- Esta reflexión está basada en las experiencias prácticas que los y las autoras del artículo están llevando a cabo en la actualidad y sus adaptaciones a los tiempos de crisis sociosanitaria que estamos viviendo a raíz de la COVID-19, así como se hacen referencia a 
trabajos de otros autores/as en diferentes campos, como el Trabajo Social, la Psicología Comunitaria y la Educación.

\section{Implicaciones para la práctica y/o política}

- La utilización de la metodología PhotoVoice, en cualquiera de los ámbitos en los que se utilice (Intervención, Educación, Cooperación, etc.), favorece procesos de toma de conciencia, empoderamiento de las personas participantes y promueve la transformación social.

\section{PHOTOVOICE: ALGUNAS CLAVES HISTÓRICAS, TEÓRICO-FILOSÓFICAS Y ÉTICAS}

La metodología que actualmente conocemos como PhotoVoice fue desarrollada e inicialmente sistematizada por las investigadoras Caroline Wang y Mary Ann Burris durante la década de los 90. Surgió como parte de la evaluación de políticas de salud pública en la esfera de la cooperación internacional. Durante la citada década fue empleada como una herramienta participativa para evaluar las necesidades, los actores y los recursos de una comunidad y abogar por cambios en las políticas y los sistemas públicos. PhotoVoice tiene sus raíces en las tradiciones teóricas y epistemológicas del empoderamiento y la educación emancipadora, la teoría feminista y la fotografía documental (Wang y Burris, 1994, 1997). Basada en la educación de Freire (1970) para la conciencia crítica (conscientizacão), la metodología PhotoVoice sitúa a los individuos y a las comunidades en el centro del descubrimiento y el análisis de un fenómeno social que les afecta y frente al que sería deseable una mayor consciencia o iniciar un proceso de conocimiento y transformación. Su enfoque teórico se nutre también de las teorías feministas, reconociendo a las mujeres (y a otros grupos marginados) como autoridades y creadoras legítimas de conocimiento (Maguire, 1987). De esta forma, siguiendo a Palibroda, Krieg, Murdock, y Havelock (2009) podemos describir PhotoVoice como un método de investigación-acción participativa que emplea la fotografía y el diálogo grupal como medio para que las personas profundicen en la comprensión de un problema o de una preocupación de la comunidad. Las imágenes visuales y las historias que las acompañan son las herramientas utilizadas para fomentar el diálogo y la reflexión grupal, además de constituir un medio para acceder a los encargados de la formulación de políticas y de la toma de decisiones dentro de la comunidad, con la finalidad de alcanzar una solución a dicho problema o preocupación. La metodología PhotoVoice es también una forma de fotografía documental ciudadana destinada a registrar y analizar problemas comunitarios y sociales con el objetivo de abogar por el cambio, por la transformación social y la búsqueda de la justicia.

De acuerdo con las tradiciones teóricas y epistemológicas feministas y freirianas, a partir del empleo de PhotoVoice se rechaza la idea de un conocimiento "objetivo" libre de valores. Al entregar una cámara a las mismas personas afectadas por el tema social que se investiga, y crear un espacio para que los y las participantes reflexionen y narren sus experiencias, promovemos la producción de conocimiento participativo, colectivo y reflexivo. A través de discusiones dialécticas, los y las participantes conectan sus experiencias personales con las estructuras y los procesos sociopolíticos, examinan críticamente los desafíos a los que se enfrentan tanto individual como colectivamente; analizan sus causas fundamentales, los factores contribuyentes, las consecuencias, y formulan los pasos necesarios para mejorar las condiciones de su comunidad. Este tipo de creación colectiva del conocimiento, además de romper con el monopolio del conocimiento "experto" (Freire, 1970; Hall, Gillette, y Tandon, 1977), motiva a los miembros de la comunidad a tomar medidas para mejorar las condiciones en las que viven, contribuyendo de esta forma tanto al empoderamiento como al fortalecimiento 
de la identidad comunitaria.

Así, la metodología PhotoVoice permite generar un proceso que hibrida tres dimensiones: investigación social y creación de conocimiento; educación y desarrollo de capacidades; y acción social para el cambio. Estas tres dimensiones se conectan directamente con los tres objetivos definidos por las autoras al sistematizar la metodología (Wang y Burris, 1997):

1. Empoderar a las personas para que documenten y reflejen las fortalezas y debilidades de su comunidad, a través de la acción de fotografiar la vida cotidiana;

2. Promover el diálogo y el conocimiento críticos, facilitando la comunicación y el diálogo mediante el análisis de grupos de fotografías, para identificar problemas comunitarios importantes;

3. Llamar la atención de los legisladores y otras personas influyentes sobre la importancia del cambio en la comunidad.

La metodología PhotoVoice es flexible y aunque se originó en el campo de la salud pública, PhotoVoice se ha utilizado en una amplia variedad de campos disciplinarios como un método participativo para examinar problemas sociales, exponer la injusticia y promover acciones para mejorar las condiciones sociales (Bananuka y John, 2015; Beh, Bruyere, y Lolosoli, 2013; Bell, 2015; Castleden, Garvin, y Nation, 2008; Catalani y Minkler, 2010; Desyllas, 2014; Hergenrather, Rhodes, Cowan, Bardhoshi, y Pula, 2009; Rhodes, Hergenrather, Wilkin, y Jolly, 2008).

La materialización práctica de un proceso de PhotoVoice implica una serie de sesiones de formación y debates sobre la metodología del proyecto, técnica fotográfica, ética del proceso para, posteriormente, abrir un periodo de toma de fotografías en base a una pregunta de investigación que apelará directamente a la vivencia de la persona vinculada a una experiencia o necesidad sentida. La pregunta será formulada por el equipo dinamizador/investigador y será contestada a través de las fotografías realizadas por las personas participantes. Es importante que la pregunta de investigación cumpla con las siguientes características:

1. Que sea una pregunta personal (que incluya "para ti”. "para usted”,"tú",etc)

2. Que sea una pregunta abierta (no contestable con "sí" o "no")

3. Que sea específica; es decir, que no sea susceptible de ser malinterpretada.

4. Que esté directamente relacionada con la vida/vivencia de las personas participantes.

Algunos ejemplos de pregunta de investigación/dinamización de este proceso pueden ser: "Según tu propia experiencia, ¿qué cambios has notado en tu comunidad desde que el proyecto X comenzó a funcionar?” o, “¿cómo ha afectado a tu vida el proyecto $\mathrm{X}$ ?"

Formulada la pregunta, tendrá lugar la primera fase de producción fotográfica en la que las personas participantes toman fotografías tanto de condiciones individuales como de la comunidad. En una segunda fase, los participantes traen las fotografías acompañadas de un breve texto explicativo-descriptivo y discuten sus experiencias y observaciones en un grupo pequeño. Así, los participantes crean "voces" a través de reflexiones continuas y discusiones dialécticas a las que además, pueden añadir textos escritos de forma breve para acompañar las fotografías seleccionadas. También es posible que el equipo técnico/investigador realice un análisis previo de las fotografías tomadas por los participantes para sugerir un orden a la hora de establecer el debate; no obstante, es importante recordar que son los participantes quienes deben tomar sus 
propias decisiones. Tanto sus fotografías como las voces que estas generan a partir del debate colectivo se difunden, posteriormente, de diversas formas. Como elemento final, tras los análisis grupales y técnicos, muchos proyectos comunitarios organizan exhibiciones para crear un espacio en el que miembros de la comunidad, legisladores y otras personas relevantes aprenden y discuten acerca del problema social que el proyecto se propuso abordar.

Actualmente, debido a las condiciones generadas por la Covid-19, especialmente relacionadas con el distanciamiento social requerido por las medidas sanitarias implementadas, muchas de las fases que contempla un proceso PhotoVoice tienden a pasar a un formato digital. Esto modifica la experiencia de los y las participantes, incluyendo particularidades que la diferencian de la metodología de PhotoVoice clásica, por lo que en otros trabajos la hemos denominado como Digital PhotoVoice (Marques et al., en prensa).

La transición de un modelo analógico de PhotoVoice a un formato digital, es particularmente fácil para los jóvenes debido a su familiaridad con las tecnologías digitales, en cuanto que ya utilizan las fotos de forma regular en las redes sociales, blogs, vlogs, etc. (Volpe, 2019). De acuerdo con esta autora, el modelo digital permite más posibilidades como, por ejemplo, su combinación con diarios digitales. Dicha combinación "es una fantástica oportunidad para interactuar con los jóvenes más allá de la forma tradicional de PhotoVoice y puede incluir vídeos, transmisiones en vivo, blogs, vlogs, juegos en consolas, aplicaciones telefónicas, correos electrónicos y mensajes" (Volpe, 2019). Esto amplía las posibilidades de identificación de los y las jóvenes con el lenguaje audiovisual empleado en la aplicación de la metodología, favoreciendo, al mismo tiempo, la participación juvenil en poblaciones digitalmente alfabetizadas.

En cuanto a las cuestiones éticas que se deben tener en cuenta a la hora de iniciar cualquier proceso de investigación social y, específicamente durante todo el proceso y desarrollo de esta metodología, autores y autoras como Creighton et al. (2018); EvansAgnew y Rosemberg (2016); Teti (2019); Wang y Redwood-Jones (2001), entre otras, han debatido ampliamente sobre las cuestiones éticas ligadas a los procesos implicados en PhotoVoice. En este sentido, siguiendo las propuestas de Liebenberg (2018); Rosemberg y Evans-Agnew (2020), se deben tener en cuenta los siguientes 4 puntos de referencia éticos:

1. La necesidad de obtener consentimientos explícitos de forma oral y escrita de todas las partes involucradas (investigadores, participantes, fotógrafos y personas a la que se va a fotografiar);

2. La importancia de clarificar tanto la o las preguntas de investigación, como los procesos, el protocolo y el plan de difusión previsto;

3. La necesidad de incorporar mecanismos de autorreflexión de forma continua durante la implementación de la metodología en la que participen todas las partes involucradas, especialmente el o la investigadora;

4. La importancia de garantizar que tanto el conocimiento como la experiencia de la comunidad será cuidado y honrado durante todo el proceso de implementación de la metodología.

Las cuestiones éticas sobre la toma de fotografías se han vuelto aún más críticas con el avance de las tecnologías digitales. Debemos tener en cuenta la protección de los y las participantes, tanto a los fotógrafos (los que participan en este proyecto) como a las personas que aparecen en las fotografías (los fotografiados). Esta protección involucra la necesidad de un consentimiento informado, una garantía de derecho a la seguridad, la privacidad y la posibilidad de abandonar el proyecto o el proceso cuando lo deseen. 
Teti (2019) nos sugiere que dotemos a nuestros proyectos de la flexibilidad necesaria para adaptar la protección ética a las diferentes situaciones a las que nos enfrentamos con los procesos de PhotoVoice; más allá de dicha flexibilidad, en todos los procesos se deben respetar parámetros éticos básicos, como señalan muchos autores Liebenberg (2018); Rosemberg y Evans-Agnew (2020); Wang y Redwood-Jones (2001) entre otros. Una discusión sobre ética es indispensable en cada proyecto de PhotoVoice, desde la planificación hasta la implementación, incluida la capacitación del personal y las personas participantes, la toma de fotografías y la difusión de fotografías y voces (Wang y Burris, 1997; Yoshihama, 2021).

\section{PHOTOVOICE COMO HERRAMIENTA PARA EL DESARROLLO LOCAL COMUNITARIO Y LA CONSTRUCCIÓN DE UNA IDENTIDAD COLECTIVA CRIITICA Y PARTICIPATIVA}

Como método de Investigación-Acción Participativa, PhotoVoice ofrece múltiples posibilidades para la transformación y la investigación social. Al incorporar métodos visuales (principalmente fotografía), va más allá de las formas tradicionales de comunicación y documentación basadas en texto, permitiendo la participación de aquellos que han sido convencionalmente excluidos de la participación y/o a los que han silenciado su voz. El trabajo clásico de Wang y Burris (1994, 1997) con mujeres con un nivel de alfabetización limitado en una aldea rural china (Yunnan) o trabajos más actuales como los de Garrido y Delgado (2017) con jóvenes del campo de refugiados en Ritsona (Grecia) o el de Martínez-Guzmán, Prado-Meza, Tapia-Muro, y Tapia-González (2018) con mujeres activistas en diferentes ámbitos sociales en la provincia de Colima (México), son algunos ejemplos de ello. PhotoVoice también trasciende las barreras del idioma, como en aquellos casos en los que los investigadores no comparten el idioma que hablan los participantes. Además del trabajo de Wang y colaboradores, la experiencia con nuestros propios proyectos nos permite ser conscientes de esta fortaleza en relación a las barreras lingüísticas. Esta metodología permite un examen crítico de los problemas que enfrentan los participantes a pesar de las barreras idiomáticas. Ejemplos de ello podemos encontrarlos en un proyecto entre mujeres refugiadas en un área metropolitana urbana en los EE. UU. (Yoshihama y Carr, 2002) y en un proyecto en las Islas Azores (Portugal) con personas sin hogar, muchas de las cuales han sido deportadas de EE.UU. y su lengua principal es el inglés (Marques, 2021; Marques et al., en prensa).

En este segundo caso, dentro del proyecto en las Islas Azores, los participantes personas sin hogar- recibieron dos cámaras analógicas desechables y se siguieron los siguientes pasos: recibieron una pequeña formación sobre el proyecto, el funcionamiento de la cámara, teoría fotográfica y aspectos éticos en la toma de fotografías. Todas las fotos fueron debatidas en grupo y fue utilizado el protocolo SHOWED para análisis y debate de las fotos seleccionadas, tal y como explicaremos a continuación. Los investigadores hicieron análisis temáticos y de contenido de las fotografías y este fue devuelto al grupo para su validación. De forma colaborativa se organizó una exposición fotográfica, se hizo la presentación pública del proyecto y los resultados fueron presentados en una conferencia el Día Internacional para la Erradicación de la Pobreza.

Como ya hemos comentado, PhotoVoice implica discusiones grupales facilitadas por la producción fotográfica y se puede apoyar en herramientas como un listado de preguntas que se agrupa bajo el acrónimo en inglés "SHOWED" que incluye las preguntas: ¿Qué ves aquí?, ¿qué está pasando realmente aquí?, ¿cómo se relaciona esto con nuestras vidas?, ¿por qué existe este problema, preocupación o fortaleza?, ¿qué podemos hacer al respecto? (Wang, 1999). Estas preguntas se utilizan para facilitar el análisis crítico de lo que capturan las fotografías de los y las participantes. Si bien 
algunos proyectos están muy estructurados en torno a estas preguntas, la flexibilidad de la metodología permite utilizar un formato semiestructurado que adapte las preguntas a los temas de discusión y las condiciones y necesidades de los miembros del grupo. Es importante destacar que PhotoVoice, a través de la toma de fotografías y discusiones dialógicas repetidas, fomenta la conciencia crítica (Carlson, Engebretson, y Chamberlain, 2006; Marques, 2021; Yoshihama y Yunomae, 2018), y en paralelo es una poderosa herramienta para trabajar aspectos comunitarios, así como de creación y fortalecimiento de la identidad colectiva.

Este proceso es, en sí mismo, un potente catalizador de experiencias y proyectos de Desarrollo Comunitario en cualquier contexto. Su contribución en la toma de conciencia de las fortalezas y debilidades que pueda tener una comunidad o un territorio, facilita procesos de transformación social y sirve de diagnóstico participado y movilizador hacia la acción y el cambio. Desde una perspectiva de la dinamización comunitaria, contribuye a favorecer procesos de enfoque "Middle-Out" (Sáez, 2017), espacios de encuentro entre los proyectos surgidos desde la Administración Pública ("Top-Down") con cargo a financiaciones públicas- de gran envergadura, pero de poco apoyo por parte de la base social- y aquellos iniciados desde la base social ("Bottom-Up") sin apoyo inicial por parte de la administración, con presupuestos muy limitados y limitantes para una mayor escalabilidad y repercusión en los procesos de transformación. Si, además, tenemos en cuenta el último de los elementos que sugiere la metodología: una exposición pública de las fotos realizadas sobre el problema investigado, el efecto multiplicador del impacto es aún mayor y su contribución a la concienciación de las autoridades competentes es de incalculable valor para la creación de políticas públicas inclusivas.

Aprovechando estas fortalezas de PhotoVoice, esta metodología es empleada en la investigación social de diferentes campos disciplinares como el Trabajo Social, la Psicología Comunitaria, la Sociología y la Educación, abarcando una amplia gama de áreas temáticas y grupos de población en todo el mundo en diversas ubicaciones / regiones / países, especialmente en proyectos destinados a empoderar a quienes han sido marginados (Desyllas, 2014; Garrido y Delgado, 2017; Lightfoot et al., 2017; Marques, 2021; Martínez-Guzmán et al., 2018; Yoshihama, 2019; Yoshihama y Carr, 2002; Yoshihama y Yunomae, 2018).

En este sentido, tal y como recogen Acebes y Delgado (2012) al hablar de los principios del Trabajo Social Comunitario, PhotoVoice favorece la intencionalidad hacia la transformación de la realidad social a partir de un conocimiento profundo y experimentado de la misma, desde una responsabilidad compartida en un proceso participativo y equitativo.

Asimismo, la versatilidad de esta herramienta metodológica para formar parte de cualquier momento de la implementación de un proyecto la convierte en una herramienta de gran utilidad en cualquier estrategia de desarrollo local comunitario que cuente con la "Ciencia Ciudadana" como referente. Cooper (2018) defiende que "los científicos ciudadanos participan en la ciencia al poner en práctica sus diferentes aficiones o preocupaciones, y no necesariamente por ser su profesión". La participación ciudadana puede contribuir en la construcción de conocimientos que ayudan a la indagación y resolución de problemas. En el contexto español, podemos citar como ejemplo el caso del proyecto "Photovoice Villaverde" que fue utilizado dentro del marco del proyecto europeo "Heart Healthy Hoods" centrado en la alimentación y la salud dentro del entorno urbano ${ }^{1}$. En este proyecto, así como ocurre en las evaluaciones posteriores de la mayoría de los procesos que implican la metodología de

\footnotetext{
${ }^{1}$ Más información sobre el proyecto en el enlace: http://hhhproject.eu/ https://www.youtube.com/watch?v=ZaXip_1Lj1Y\&ab_channel=SocialandCardiovascularEpidemiology (Última visita: 13/10/2021)
} 
PhotoVoice, las personas participantes declararon ser más conscientes de las prácticas individuales y colectivas en torno a las temáticas y problemas que se trabajan y se explicitan. Como resultados del mencionado proyecto de salud, podemos destacar las declaraciones de algunos participantes que alegaban que, gracias a haber utilizado la lente de una cámara para mirar la realidad, ahora eran más conscientes de la cantidad de gente sin recursos que busca comida por las calles y que dicha consciencia les había hecho más sensibles y proactivos a la hora de aportar y/o ayudar a la prevención de situaciones de riesgo. Otros participantes, por ejemplo, argumentaban cómo el propio proceso les había permitido extender su red de amigos y conocidos dentro de la propia comunidad.

La utilización de las entrevistas semi-estructuradas, la observación de los propios grupos de trabajo, así como los cuestionarios, previos y posteriores al proceso, permiten la evaluación de estos cambios y del empoderamiento de las personas participantes en estos procesos.

Estos son algunos de los motivos por los que la herramienta PhotoVoice, además, está dentro de los programas formativos en Trabajo Social (Mulder, 2014; Mulder y Dull, 2014; Peabody, 2013) y cada vez más presente en los vinculados a los métodos de investigación en Sociología (Manohar, Berkowitz, Wilder, y Tinkler, 2012; Schell, Ferguson, Hamoline, Shea, y Thomas-Maclean, 2009), Psicología Comunitaria (Guariso, Paloma, Arias, Garrido, y García-Ramírez, 2016; Migliorini y Rania, 2017), así como en disciplinas vinculadas a la formación de profesorado en facultades de Educación, como tendremos ocasión de profundizar en apartados posteriores al hablar del proyecto Erasmus + "ESCUTA" (2020-2023).

\section{PHOTOVOICE EN LA ERA DIGITAL}

La tecnología de la información y la comunicación (TIC) se ha convertido en un importante motor de cambio en el mundo. Una nueva generación de teléfonos inteligentes reúne múltiples formas de comunicación y la posibilidad de emplear miles de aplicaciones que afectan la forma en que vivimos e interaccionamos con los demás al tiempo que redefinen de manera continua la relación entre sociedad, cultura y tecnología. En este artículo, utilizamos el término "digital" vinculándolo a los procesos de investigación tal y como lo emplea Dawson "para describir los métodos de investigación que utilizan productos y soluciones informáticas (o tecnologías electrónicas) para la recopilación y el análisis de datos" (2020, p. 1). El mundo digital abre una amplia gama de nuevas posibilidades para PhotoVoice, desde el uso de cámaras digitales y teléfonos móviles como herramienta de autoexpresión, documentación y reflexión crítica- hasta el uso de plataformas digitales para difundir fotografías y voces de manera amplia y creativa. La difusión de las tecnologías digitales ha ampliado la diversidad de instrumentos utilizados en la investigación y la intervención en Ciencias Sociales.

Así como se han adaptado otras metodologías y enfoques al mundo digital Visualización de datos, Etnografía digital, Storytelling digital, Métodos visuales digitales, Diarios móviles, Herramientas de colaboración online, Grupos focales online, Observación online, Comunidades de investigación online, entre otros-, creemos que la aplicación digital de PhotoVoice es necesaria y de fácil acceso.

Dado que la fotografía constituye el núcleo central de PhotoVoice, la reciente transición rápida de la fotografía analógica a la fotografía digital, junto con la disponibilidad masiva de teléfonos inteligentes que incorporan una cámara, ahora permite que muchas personas tengan acceso a la fotografía. Schankweiler, Straub, y Wendl explican que: "las nuevas tecnologías han permitido a las personas grabar, cargar y compartir imágenes directamente a través de dispositivos móviles, lo que convierte a casi todo el mundo en un testigo potencial en un momento dado" (2019, p. 1). La fotografía digital es una forma versátil y accesible de expresión creativa y ofrece oportunidades de 
participación, ejercicio de la ciudadanía y una inclusión verdaderamente significativa que pocas formas artísticas pueden hacer. La fotografía digital permite a las personas compartir sus perspectivas y experiencias o crear un espacio para la comunicación y la discusión de una manera que sea instantáneamente atractiva y accesible para todos. Además, debido a que los avances tecnológicos en la fotografía digital permiten mejorar la calidad de una fotografía (por ejemplo, ajustar el contraste, el brillo o el color), los participantes pueden enfocarse más en ideas, problemas y fortalezas, y dedicar menos atención al aspecto técnico de la fotografía.

Además de la fotografía digital, la mayor disponibilidad de redes sociales y plataformas digitales enriquece los proyectos que utilizan PhotoVoice. Según Olsson (2014), "el nuevo entorno mediático, construido sobre la lógica de la "web 2.0", ofrece posibilidades participativas nuevas y hasta ahora inéditas y, como tal, puede convertirse en una arquitectura participativa para los ciudadanos". Este elemento participativo concuerda con los principios de PhotoVoice y puede aumentar su impacto debido a que las redes sociales brindan una plataforma interactiva y actualizada para la participación social activa, lo que ha transformado la forma de enviar y recibir información. A pesar de ser conscientes y compartir la preocupación por los riesgos potenciales de las nuevas tecnologías en fenómenos como el ciberacoso (Fajardo-Bullón et al., 2021) o las socioadicciones (González et al., 2016), nos alineamos con autores como Mcnutt (2018), que creen que un uso adecuado de las mismas contribuye a complementar a los métodos más tradicionales y ayudan al esfuerzo por generar un cambio social, haciéndolo más productivo y exitoso.

En el caso del PhotoVoice Digital y vinculado a la producción de imágenes, en la actualidad existen redes sociales como Instagram, Twitch, Tik-Tok, Flicker, Facebook, Twitter, etc., que permiten a las personas con un teléfono inteligente (smartphone) contribuir a la producción de imágenes que, con una utilización bien estructurada, éticamente adecuada y alejada de los potenciales riesgos que hemos citado, prestan un servicio incalculable a la transformación social, especialmente en las generaciones más jóvenes y más socializadas en el lenguaje audiovisual.

En este sentido, la fotografía y las plataformas digitales pueden facilitar la construcción de relaciones y contribuir a fortalecer procesos de participación. Autores como Reamer (2013) afirman que el uso de los medios digitales puede favorecer que las personas que trabajan en intervención socio-sanitaria puedan ser más accesibles y humanizar las relaciones entre ellas y la población, especialmente en situaciones en las que el contacto directo personal no es posible, véase el caso de situaciones de movilidad reducida, orografía complicada o las medidas de confinamiento impuestas por la situación pandémica de la Covid-19.

Así, los medios digitales pueden ayudar a proporcionar apoyo social, nuevas formas de relación y ofrecernos nuevas herramientas para la dinamización. Chen (2012), en su libro 50 Digital Team-Building Games, enumera dinámicas para romper el hielo, actividades grupales utilizando redes sociales, teléfonos inteligentes, GPS, tablets, etc. En su libro, la fotografía se utiliza en varios ejercicios como "Photo Booth -IPad 2 o redes basadas en fotografías", "GooseChase: búsqueda del tesoro de fotos con teléfonos celulares" o "Digital Slideshow-Picture Your Success". Estas actividades, entre otras, se pueden incorporar a Digital Photovoice, enriquecer su aplicación y, además, contribuir a la construcción de la identidad colectiva y al fortalecimiento de la cohesión grupal, cuestiones sobre las que incidiremos en el siguiente apartado.

\section{4. (DIGITAL) PHOTOVOICE Y LA CONSTRUCCIÓN DE LA IDENTIDAD COLECTIVA: DE LA CREACIÓN AL FORTALECIMIENTO DE LA COMUNIDAD}

El enfoque de la psicología sociocultural guía nuestra perspectiva sobre el concepto de identidad que manejamos en nuestro trabajo. En este sentido, la teoría de la acción 
mediada desarrollada en el ya clásico trabajo de Penuel y Wertsch (1995) se aborda el estudio de la identidad tomando en cuenta, primero, los contextos en los que la identidad de los individuos va tomando forma, gracias a la participación de los mismos en actividades concretas y, segundo, los recursos culturales disponibles en un momento histórico puntual, los cuales favorecen o restringen dichas construcciones identitarias. De esta forma, entendemos la identidad como un proceso dinámico en el que se establece un sentido de pertenencia -individual y colectivo- que se da siempre en interacción con otros, con los que se negocian significados y posicionamientos, dentro de procesos dialógicos (Bajtin, 1981). La identidad, como fenómeno coconstruido, por tanto, se conceptualiza como un fenómeno fluido, híbrido y cambiante en función de las interacciones y de las herramientas disponibles en los contextos sociales y momentos históricos en los que las interacciones tienen lugar. Siguiendo este enfoque, el empleo de la fotografía digital, como mediador en la experiencia de construcción identitaria de los y las participantes, posibilita procesos de autorreflexión y empáticos que abordaremos a continuación.

El empleo de PhotoVoice favorece tanto la autorreflexión, como la co-construcción de relatos y el acceso a perspectivas diferentes sobre un fenómeno concreto que pueden diferir de las propias, propiciando oportunidades únicas para aprender a mirar dicho fenómeno o problema social desde otro lugar. Esto, acompañado de la discusión y análisis colectivo del material visual producido, favorece la aparición de voces (genéricas, ideológicas, institucionales...) que los y las participantes pueden asumir, reformular o rechazar, enriqueciendo el proceso dialógico en el se ven imbuidos los y las participantes. PhotoVoice, tanto en su versión presencial, como en su versión digital, contribuye tanto a la construcción identitaria de la comunidad cuando los lazos de unión son todavía difusos, como al fortalecimiento de la misma cuando los y las participantes ya se identifican previamente con el grupo y con los espacios que habitan.

En este sentido, existen proyectos actuales en los que (Digital) PhotoVoice está contribuyendo en el objetivo de la co-construcción de los espacios urbanos inclusivos y sostenibles a partir de la participación ciudadana y la construcción de una identidad colectiva vinculada a ese territorio. Así, en el proyecto europeo URBINAT (H2020) ${ }^{2}$ el empleo de PhotoVoice dentro de las estrategias de diagnóstico participado -en las ciudades europeas de Oporto, Nantes y Sofía-, ha permitido no sólo identificar los espacios con los que la ciudadanía se siente más vinculada o aquellos que le gustaría transformar, sino que ha favorecido la proyección de alternativas de planificación urbanística futuras que, en este caso, se van a realizar a través de Nature-Based Solutions $(\mathrm{NBS})^{3}$.

\section{5. (DIGITAL) PHOTOVOICE EN EL ÁMBITO EDUCATIVO}

Existen muchas experiencias del uso de la fotografía en el entorno educativo. No debemos olvidar proyectos pioneros como "Cameras in the Curriculum" (Ford, 1982) desarrollado entre 1982 y 1983 en los EEUU y en cuya publicación se describen 119 proyectos planificados y desarrollados por maestros que utilizan la fotografía como parte integral del plan de estudios con el patrocinio de Kodak. Era una modalidad diferente a PhotoVoice pero forma parte de la génesis del uso de la fotografía en los espacios educativos, además de ser un referente a nivel histórico en esta área.

Existen otras referencias más actuales en el uso de la cámara en las aulas y de PhotoVoice con fines educativos (Barrett-Dragan, 2008; Shah, 2014) e, incluso, manua-

\footnotetext{
${ }^{2}$ Más información del proyecto URBINAT en: https://urbinat.eu/ (Última visita: 13/10/2021)

${ }^{3}$ Para ampliar la información sobre las Nature-Based Solutions, visitar el enlace: https://urbinat.eu/nbs-c atalogue/
} 
les prácticos y actuales como el desarrollado por Latz (2017), que nos orientan en el proceso de diseñar un proyecto de PhotoVoice, señalando tanto las posibilidades de análisis de los datos y la información generada en el proceso, como orientaciones para la exposición pública de las fotografías creadas durante el mismo.

Vinculado a los espacios educativos, y motivado por la actual crisis sociosanitaria por la Covid-19, en noviembre de 2020 desarrollamos una experiencia formativa-experiencial de PhotoVoice en un centro educativo de la ciudad de Cáceres (Extremadura, España) en la que participó alumnado de los ciclos formativos en integración social y promoción de la igualdad del CEIP Al-Qazeres FernándezPacheco et al. (en prensa). Nuestra pregunta de investigación, y objetivo de la práctica, se centraba en explorar e intentar comprender qué había cambiado en la vida del alumnado con motivo de la COVID-19, realizar un análisis de manera participada y explorar las vivencias del alumnado vinculadas a espacios familiares, de ocio y dentro del ámbito educativo. Las restricciones de contacto social establecidas en ese momento por las autoridades sanitarias presentaron un auténtico desafío. La implementación del PhotoVoice en este caso se convirtió en un modelo híbrido entre la presencialidad y la virtualidad. En los análisis realizados, tanto grupalmente como a nivel técnico-investigador, encontramos diferentes aproximaciones discursivas frente a la experiencia vivida articulada en torno a dos ejes: un eje discursivo centrado en la pérdida ("lo que la Covid-19 les ha hecho perder" o "les ha quitado", por ejemplo: la posibilidad de reunirse de forma presencial con grupos extensos de amigos; los abrazos y los besos a las personas queridas, especialmente a sus mayores, etc.); y otro eje que aglutina los discursos centrados en el cambio ("lo que la Covid-19 ha modificado"), en los que encontramos tanto elementos positivos (i.e.: mayor unión con la familia nuclear; la adquisición de nuevas competencias digitales), como negativos (i.e.: la incorporación de protocolos sanitarios para posibilitar el contacto social -uso de geles hidroalcohólicos, mascarillas, etc.-) (Fernández-Pacheco et al., en prensa).

Los espacios de análisis grupal se convirtieron en espacios de ejercicio de la empatía y empoderamiento en el que el alumnado pudo expresar sus vivencias durante el confinamiento, como la transformación de las prácticas educativas durante el mismo, al tiempo que pudieron repensar conjuntamente los significados de la "nueva normalidad" y expresar su inconformidad frente al etiquetado de "juventud irresponsable" por parte de algunos medios de comunicación. De esta forma, se creó un estrechamiento de lazos grupales, fortaleciendo su identidad colectiva y favoreciendo discursos desde una perspectiva del "nosotros" frente al individualismo derivado de la distancia social y el miedo al contagio.

Otra experiencia reciente en el empleo de PhotoVoice en el ámbito educativo, se encuentra vinculada a un proyecto Erasmus+ bajo el acrónimo ESCUTA ${ }^{4}$ (Emprendimiento Social Comunitario Universitario Transnacional - Açores), que actualmente estamos desarrollando y que tiene por objetivo el aprendizaje de competencias SocioEmocionales (SEL) por parte del alumnado universitario a partir de su participación en talleres formativos y el diagnóstico e implementación de un proyecto de desarrollo comunitario. El aprendizaje social y emocional según Johnson y Johnson (2004) consiste en el:

- Dominio y uso apropiado de las habilidades interpersonales y de grupos pequeños (por ejemplo, reconocer, manejar y expresar apropiadamente las propias emociones) y,

- La integración interna de actitudes y valores prosociales necesarios para alcanzar objetivos, resolver problemas, involucrarse emocionalmente en el aprendizaje y el trabajo, y tener éxito en la escuela y durante toda la vida.

\footnotetext{
${ }^{4}$ Se puede visitar su página web en el siguiente enlace: https://escuta.com.pt/
} 
El proyecto ESCUTA, siguiendo estos principios, pretende favorecer durante su desarrollo (2020-2023) el aprendizaje de las "Cinco Grandes" habilidades sociales y emocionales:

1. La apertura a la experiencia (apertura mental)

2. La conciencia (desempeño de tareas)

3. La estabilidad emocional (regulación emocional)

4. La extraversión (interacción con otros) y

5. La amabilidad (colaboración)

Para lograr este objetivo, los estudiantes que forman parte de este proyecto están recibiendo formación en varios métodos participativos, entre ellos PhotoVoice. Han experimentado sesiones de esta técnica y van a desarrollar dentro de su comunidad procesos vinculados a cuestiones relacionadas con la Economía, la Sostenibilidad y la Exclusión Social. Este proyecto hibrida prácticas de PhotoVoice presenciales y digitales, así como con otras herramientas de la Investigación-Acción Participativa (IAP) con el objetivo de reflexionar, diagnosticar y proponer alternativas a los problemas de las comunidades en las que, posteriormente, se implementarán dos proyectos de Desarrollo Comunitario desde el enfoque de la Economía Circular, Social y Solidaria. En este momento nos encontramos en plena implementación de estas formaciones con estudiantes de la Universidad de Extremadura en el campus de Cáceres pero, podemos adelantar algunos de los resultados obtenidos con el alumnado de la Universidad dos Açores, de cuya experiencia nos gustaría destacar el desarrollo tanto de habilidades socio-emocionales como de pensamiento crítico y reflexivo. Tras la experiencia PhotoVoice con el alumnado del proyecto ESCUTA, se llevó a cabo una evaluación a través de cuestionarios y entrevistas grupales en la que quedó patente que las reflexiones suscitadas del proceso de producción fotográfica, así como de la reflexión grupal se produjo un aumento de la conciencia social en torno a los temas planteados (Economía, Desarrollo y Sostenibilidad). En dicho proceso pudimos comprobar, asimismo, un aumento del "autoconocimiento personal" del alumnado participante a través de una reflexión crítica sobre sus propias prácticas vinculadas a las temáticas mencionadas ${ }^{5}$.

Pimienta (2012) defiende que "la clave del aprendizaje significativo radica en relacionar el nuevo material con las ideas ya existentes en la estructura cognitiva del estudiante. Por consiguiente, la eficacia de tal aprendizaje está en función de su carácter significativo, y no en las técnicas memorísticas" (Pimienta, 2012, p.3). En el contexto del proyecto ESCUTA entendemos las competencias como "las actuaciones que tienen las personas para resolver problemas integrales del contexto, con ética, idoneidad, apropiación del conocimiento y puesta en acción de las habilidades necesarias" (Tobón, Pimienta, y Fraile, 2010). La utilización del PhotoVoice, en este tipo de proyectos no sólo favorece en el alumnado su comunicación visual y la adquisición de otras competencias tales como la digital, sino que, al mismo tiempo, el empleo de estrategias metodológicas que emplean la fotografía como es el caso de PhotoVoice, aumenta enormemente las posibilidades de la investigación y la acción social dentro del ámbito educativo.

\section{DIGITAL PHOTOVOICE: ALGUNAS REFLEXIONES FINALES Y UN CAMINO HACIA LA INVESTIGACIÓN E INTERVENCIÓN DIGITAL}

Desde los inicios de la modernidad, la tecnología ha transformado las escalas (GlobalLocal) y las formas en la que actuamos e interactuamos en sociedad, así como nuestra

\footnotetext{
${ }^{5}$ Los resultados de las evaluaciones, así como otros contenidos vinculados al proyecto, estarán disponibles en la web del proyecto ESCUTA: https://escuta.com.pt/ (Última visita: 129/12/2021)
} 
relación con los entornos en los que habitamos y de los que somos interdependientes. A medida que avanzamos en la utilización de nuevas tecnologías surgen espacios para la innovación, para las dudas y para los debates éticos que son necesarios afrontar ligados a la nueva tecnología.

En este sentido, y para el artículo que nos ocupa, nos hemos centrado en la descripción y análisis de una metodología, (Digital) PhotoVoice, que, partiendo de la Investigación-Acción Participativa, busca la interrelación personal, la construcción y fortalecimiento de la identidad colectiva y la dinamización comunitaria para fomentar acciones colectivas frente a la injusticia -económica, social-y la vulnerabilidad socio-ambiental. El papel de esta metodología cobra un nuevo sentido en su adaptación a la sociedad digital y a su aplicación utilizando nuevas herramientas digitales, así como el abanico de posibilidades que ofrece la red 2.0.

Como toda nueva aplicación que se vincula a procesos de interrelación entre personas, bien sea como investigadores o como profesionales de la intervención psicosocial, es necesario un debate ético sobre cómo utilizarla, máxime teniendo en cuenta la exposición mediática a la que nos sometemos en la utilización de las redes, pero siendo conscientes de las oportunidades que suponen estas como espacios de innovación, de comunicación y de transformación social. Se necesitan procedimientos éticos para que todo lo que se haga se entienda y obtenga el acuerdo de todos los participantes, pero la exposición de las personas y sus voces tiene sus riesgos. Las imágenes y los textos cargados se vuelven visibles para un número indeterminado de personas y permanecen en la red de forma indefinida. Si bien existen formas de restringir el acceso, esto conlleva una exposición y difusión limitadas de la información que precisamente se quería hacer llegar a la sociedad a través de las fotografías y las voces que cada una de ellas representan. Sopesar los beneficios de una amplia difusión y el riesgo de una exposición excesiva (y no deseada) puede resultar difícil, pero es uno de los retos que tenemos por delante.

La flexibilidad que nos otorga PhotoVoice, tanto en su versión analógica, como digital, favorece, como ya hemos mencionado, que podamos adaptar esta metodología a diferentes contextos y a diferentes colectivos implicados en procesos de desarrollo comunitario. Sin importar el grado de destreza tecnológica, PhotoVoice permite integrar tanto a personas mayores, con menor dominio de la tecnología digital, como a jóvenes habituados al mundo digital y sus herramientas. Asimismo, PhotoVoice permite que una gran cantidad de colectivos de mayor o menor vulnerabilidad puedan formar parte de procesos de diagnóstico y/o evaluación participada en sus propios territorios y comunidades, como queda demostrado no sólo por las experiencias expuestas en este artículo, sino por la gran cantidad de literatura y de prácticas citadas en el mismo.

Teniendo en cuenta todas estas consideraciones y siendo conscientes de los retos éticos que ya hemos mencionado a lo largo de este trabajo, consideramos que (Digital) PhotoVoice constituye una metodología especialmente interesante que permite superar barreras (físicas, geográficas, lingüísticas) al tiempo que contribuye con elementos innovadores a la investigación social e intervención socio-comunitaria con grandes aportes dentro de campos disciplinarios como el Trabajo Social, la Psicología Comunitaria, la Sociología y la Educación.

\section{REFERENCIAS}

Acebes, R., y Delgado, L. (2012). Modelos teóricos y campos de intervención en el ámbito comunitario. Reflexiones sobre una experiencia de trabajo social comunitario en España. En M. del Fresno, S. Segado, y A. López (Eds.), Trabajo Social con Comunidades en el siglo XXI. Madrid: Universitas.

Bajtin, M. (1981). The dialogic imagination. En M. Holquist (Ed.), Four essays by M.M. Bakhtin. Austin: University of Texas Press. 
Bananuka, T., y John, V. M. (2015). Picturing community development work in Uganda: Fostering dialogue through Photovoice. Community Development fournal, 50(2), 196212. https://doi.org/10.1093/cdj/bsu036

Barrett-Dragan, P. (2008). Kids, Cameras, and the Curriculum. Portsmouth, NH: Heinemann. Beh, A., Bruyere, B. L., y Lolosoli, S. (2013). Legitimizing local perspectives in conservation through community-based research: A Photovoice study in Samburu, Kenya. Society and Natural Resources, 26(12), 1390-1406. https://doi.org/10.1080/08941920.2013.805858

Bell, S. E. (2015). Bridging activism and the academy: Exposing environmental injustices through the feminist ethnographic method of Photovoice. Human Ecology Review, 21(1), 27-58. http://dx.doi.org/10.22459/HER.21.01.2015.02

Carlson, E. D., Engebretson, J., y Chamberlain, R. M. (2006). Photovoice as a social process of critical consciousness. Qualitative Health Research, 16, 836-852. Descargado de https://journals.sagepub.com/doi/pdf/10.1177/1049732306287525

Castleden, H., Garvin, T., y Nation, H. F. (2008). Modifying Photovoice for communitybased participatory Indigenous research. Social Science \& Medicine, 66(6), 1393-1405. https://doi.org/10.1016/j.socscimed.2007.11.030

Catalani, C., y Minkler, M. (2010). Photovoice: A review of the literature in health and public health. Health Education \& Behavior, 37(3), 424-451. https://doi.org/10.1177/ 1090198109342084

Chen, J. (2012). 50 digital team-building games: Fast, fun meeting openers, group activities and adventures using social media, smart phones, GPS, tablets, and more. Wiley \& Sons.

Cooper, C. (2018). Ciencia ciudadana. Cómo podemos todos contribuir al conocimiento científico. Libros Grano de Sal.

Creighton, G., Oliffe, J. L., Ferlatte, O., Bottorff, J., Broom, A., y Jenkins, E. K. (2018). Photovoice ethics: Critical reflections from men's mental health research. Qualitative Health Research, 28(3), 446-455. http://dx.doi.org/10.1177/1049732317729137

Dawson, C. (2020). A-Z of digital research methods. Routledge.

Desyllas, M. C. (2014). Using photovoice with sex workers: The power of art, agency and resistance. Qualitative Social Work, 13(4), 477-501. https://doi.org/10.1177/ 1473325013496596

Evans-Agnew, R. A., y Rosemberg, M.-A. S. (2016). Questioning Photovoice research: Whose voice? Qualitative Health Research, 26(8), 1019-1030. Descargado de https://doi.org/ 10.1177/1049732315624223 https://doi.org/10.1177/1049732315624223

Fajardo-Bullón, F., Rasskin-Gutman, I., Pacheco, J. R., Barco, B. L.-D., Burguillo, B., y FelipeCastaño, E. (2021). Analysis of mental health in cyberbullying victims and perpetrators in Spanish and Colombian adolescents. Revista Latinoamericana de Psicología, 53, 122132. https://doi.org/10.14349/rlp.2021.v53.14

Fernández-Pacheco, J. L., Rasskin-Gutman, I., Marques, E., y Yoshihama, M. (en prensa). High school students and Covid -19: Feelings and dealings. A PhotoVoice experience in Extremadura. Spain.

Ford, F. (Ed.). (1982). Cameras in the Curriculum. A Challenge to Teacher Creativity. Volume 1, 1982/83. An NEA/KODAK Program. (Vol. 1). Descargado de http://files.eric.ed.gov/ fulltext/ED238409.pdf

Freire, P. (1970). Pedagogy of the oppressed. New York, NY: Continuum.

Garrido, R., y Delgado, A. (2017). La voz y la mirada de jóvenes refugiados/as en Grecia: Photovoice como herramienta de Investigación-Acción Comunitaria. Papeles de Trabajo sobre Cultura, Educación y Desarrollo Humano, 13(4), 52-65. Descargado de https://cespyd.es/publicaciones/la-voz-y-la-mirada-de-jovenes-refugiados-en-grecia -photovoice-como-herramienta-de-investigacion-accion-comunitaria/

González, V., Santamaria, J. J., López-Fernández, O., Montero, E., Merino, L., y GonzálezBueso, V. (2016). OR-34: testing problematic technological scales in socio-addict patients: the case of the mobile phone. Journal of Behavioral Addictions, 5(S1), 16-17.

Guariso, G., Paloma, V., Arias, S., Garrido, R., y García-Ramírez, M. (2016). Photovoice as a 
Research-Intervention Tool for Youth Neighborhood Activism in Societally Vulnerable Contexts. Global fournal of Community Psychology Practice, 7(3), 1-18. Descargado de https://hdl.handle.net/11441/79152

Hall, B. L., Gillette, A., y Tandon, R. (1977). Creating knowledge: Breaking the monopoly: Research methods, participation and development. International Council for Adult Education. Toronto: International Council for Adult Education.

Hergenrather, K. C., Rhodes, S. D., Cowan, C. A., Bardhoshi, G., y Pula, S. (2009). Photovoice as community-based participatory research: A qualitative review. American fournal of Health Behavior, 33(6), 686-698. http://dx.doi.org/10.5993/AJHB.33.6.6

Johnson, D., y Johnson, R. (2004). The Three Cs of promoting Social and Emotional Learning. En J. E. Zins, R. P. Weissberg, M. C. Wang, y H. J. Walberg (Eds.), Building academic success on social and emotional learning: What does the research say? Teachers College Press.

Latz, A. O. (2017). Photovoice research in education and beyond: A practical guide from theory to exhibition. London: Routledge.

Liebenberg, L. (2018). Thinking critically about Photovoice: Achieving empowerment and social change. International fournal of Qualitative Methods, 17(1). https://doi.org/ 10.1177/1609406918757631

Lightfoot, A. F., Thatcher, K., Simán, F. M., Eng, E., Merino, Y., Thomas, T., ... Chapman, M. V. (2017). "What I wish my doctor knew about my life": Using photovoice with immigrant Latino adolescents to explore barriers to healthcare. Qualitative Social Work, 18(1), 60-80. https://doi.org/10.1177/1473325017704034

Maguire, P. (1987). Doing participatory research: A feminist approach. Amherst, MA: Center for International Education, School of Education, University of Massachusetts.

Manohar, N. N., Berkowitz, D., Wilder, J., y Tinkler, J. E. (2012). Photovoice: A Critical Pedagogical Assignment in the Sociology Classroom. Currents in Teaching and Learning, 5(1\&2), 36-51.

Marques, E. (2021). A cidadania através da fotografia: a utilização do photovoice na promoção dos Direitos Humanos de pessoas em situação de sem abrigo. Revista Inclusiones, 8, 421-434. https://revistainclusiones.org/index.php/inclu/article/view/ 1024

Marques, E., Fernández-Pacheco, J. L., y Yoshihama, M. (en prensa). The right to belong and live: PhotoVoice as methodology to reflect about home as a human right.

Martínez-Guzmán, A., Prado-Meza, C. M., Tapia-Muro, C., y Tapia-González, A. (2018). Una relectura de Fotovoz como herramienta metodológica para la Investigación Social Participativa desde una perspectiva Feminista. EMPIRIA. Revista de Metodología de Ciencias Sociales, 41, 157-185. https://doi.org/10.5944/empiria.41.2018.22608

Mcnutt, J. G. (2018). Technology, activism, and social justice in a digital age. Oxford University Press.

Migliorini, L., y Rania, N. (2017). A qualitative method to "make visible" the world of intercultural relationships: the photovoice in social psychology. Qualitative Research in Psychology, 14(2), 131-145. https://doi.org/10.1080/14780887.2016.1263698

Mulder, C. (2014). Unraveling students' experiences with religion and spirituality in the classroom using a Photovoice method: Implications for MSW programs. Social Work and Christianity, 41(1), 16-44.

Mulder, C., y Dull, A. (2014). Facilitating self-reflection: The integration of Photovoice in graduate social work education. Social Work Education, 33(8), 1017-1036. https:// doi.org/10.1080/02615479.2014.937416

Olsson, T. (2014). The architecture of participation" for citizens or consumers. En C. Fuchs y S. Marisol (Eds.), Critique, social media and the information society (pp. 203-235). Routledge.

Palibroda, B., Krieg, B., Murdock, L., y Havelock, J. (2009). A practical guide to photovoice: sharing pictures, telling stories and changing communities. En and others (Ed.), 
Winnipeg, Manitoba: The Prairie Women's Health Centre of Excellence.

Peabody, C. G. (2013). Using Photovoice as a tool to engage social work students in social justice. Journal of Teaching in Social Work, 33(3), 251-265. https://doi.org/10.1080/ 08841233.2013.795922

Penuel, W. R., y Wertsch, J. V. (1995). Vygotsky and identity formation: A sociocultural approach. Educational Psychologist, 30(2), 83-92. https://doi.org/10.1207/ s15326985ep3002_5

Pimienta, J. H. (2012). Estrategias de enseñanza-aprendizaje: docencia universitaria basada en competencia. México: Pearson Educación.

Reamer, F. G. (2013). Social work in a digital age: Ethical and risk - management challenges. Social Work, 58(2), 163-172. https://doi.org/10.1093/sw/swt003

Rhodes, S. D., Hergenrather, K. C., Wilkin, A. M., y Jolly, C. (2008). Visions and voices: Indigent persons living with HIV in the Southern United States use Photovoice to create knowledge, develop partnerships, and take action. Health Promotion Practice, 9(2), 159-169. http://dx.doi.org/10.1177/1524839906293829

Rosemberg, M. A., y Evans-Agnew, R. (2020). Ethics in Photovoice: A response to Teti. International fournal of Qualitative Methods, 19. https://doi.org/10.1177/ 1609406920922734

Sáez, J. L. F.-P. (2017). (Tesis Doctoral, UCM, Madrid). Descargado de https://eprints.ucm.es/ $\mathrm{id} /$ eprint/4\{\protect \char"2026\relax\}

Schankweiler, K., Straub, V., y Wendl, T. (2019). Image testimonies: Witnessing in times of social media (K. Schankweiler, V. Straub, y T. Wendl, Eds.). London: Routledge.

Schell, K., Ferguson, A., Hamoline, R., Shea, J., y Thomas-Maclean, R. L. (2009). Photovoice as a Teaching Tool: Learning by Doing with Visual Methods. The International Journal of Teaching and Learning in Higher Education, 21, 340-352. Descargado de photovoiceasateachingtoollearningbydoi\{łprotect|char"2026\relax\}

Shah, P. P. (2014). Spaces to Speak: Photovoice and the Reimagination of Girls' Education in India. Comparative Education Review, 59(1), 50-74. Descargado de https://www .journals.uchicago.edu/doi/full/10.1086/678699

Teti, M. (2019). The murky ethics of visual qualitative methods: Picturing a clear path forward. International fournal of Qualitative Methods, 18. https://doi.org/10.1177/ 1609406919884810

Tobón, S., Pimienta, J. H., y Fraile, J. A. G. (2010). Secuencias Didácticas: Aprendizaje y evaluación de competencias. México: Pearson Educación.

Volpe, C. R. (2019). Digital diaries: new uses of PhotoVoice in participatory research with young people. Children's Geographies, 17(3), 361-370. https://doi.org/10.1080/14733285 .2018 .1543852

Wang, C. (1999). Photovoice: A participatory action research strategy applied to women's health. Journal of Women's Health, 8, 185-192. https://doi.org/10.1089/jwh.1999.8.185

Wang, C., y Burris, M. A. (1994). Empowerment through Photo Novella: Portraits of participation. Health Education \& Behavior, 21, 171-186. https://doi.org/10.1177/ 109019819402100204

Wang, C., y Burris, M. A. (1997). Photovoice: Concept, methodology, and use for participatory needs assessment. Health Education \& Behavior, 24, 369-387. Descargado de https://deepblue.lib.umich.edu/bitstream/handle/2027.42/67790/10 .1177_109019819702400309.pdf;jsessionid=EC3E4ADBB444D536179F1B2A8F0FC821 ?sequence $=2$

Wang, C., y Redwood-Jones, Y. A. (2001). Photovoice ethics: Perspectives from the Flint photovoice. Health Education \& Behavior, 28(5), 560-572. https://doi.org/10.1177/ 109019810102800504

Yoshihama, M. (2019). PhotoVoice Project: A participatory research and action in postdisaster Japan. En E. Huss y E. Bos (Eds.), Art in social work practice: Theory and practice - International perspectives (pp. 57-67). London: Routledge. 
Yoshihama, M. (2021). Visualizing drivers of gender health disparities: Ongoing participatory action research following the 2011 disaster in Japan. Social Science \& Medicine, 283. https://doi.org/10.1016/j.socscimed.2021.114133

Yoshihama, M., y Carr, E. S. (2002). Community participation reconsidered: Feminist participatory action research with Hmong women. Journal of Community Practice, 10(4), 85-103. https://doi.org/10.1300/J125v10n04_06

Yoshihama, M., y Yunomae, T. (2018). Participatory investigation of the Great East Japan Disaster: PhotoVoice from women affected by the calamity. Social Work, 63(3), 234243. http://dx.doi.org/10.1093/sw/swy018 